J. Austral. Math. Soc. Ser. B 29 (1987), 103-125

\title{
PROPERTIES OF SHORT-CRESTED WAVES IN WATER OF FINITE DEPTH
}

\author{
T. R. MARCHANT ${ }^{1}$ AND A. J. ROBERTS ${ }^{1}$
}

(Received 17 April 1986; revised 14 August 1986)

\begin{abstract}
Short-crested waves are defined as propagating surface gravity waves which are doublyperiodic in the horizontal plane. Linearly, the short-crested wave system we consider occurs when two progressive wavetrains of equal amplitude and frequency are propagating at an angle to each other.

Solutions are calculated via a computer-generated perturbation expansion in wave steepness. Harmonic resonance affects the solutions but Padé approximants can be used to estimate wave properties such as maximum wave steepness, frequency, kinetic energy and potential energy.

The force exerted by waves being reflected by a seawall is also calculated. Our results for the maximum depth-integrated onshore wave force in the standing wave limit are compared with experiment. The maximum force exerted on a seawall occurs for a steep wave in shallow water incident at an oblique angle. Results are given for this maximum force.
\end{abstract}

\section{Introduction}

Short-crested waves are defined as propagating surface gravity waves which are doubly-periodic in the horizontal plane. We shall consider the short-crested wave system found when two progressive wavetrains of equal amplitude and frequency propagate at an angle to each other. The limiting cases in this family are the less complex two-dimensional progressive waves (where the wavetrains propagate in the same direction) and two-dimensional standing waves (where the wavetrains propagate in opposite directions).

\footnotetext{
${ }^{1}$ Department of Applied Mathematics, University of Adelaide G. P. O. Box 498, Adelaide, S. A. 5001, Australia.

@ Copyright Australian Mathematical Society 1987, Serial-fee code 0334-2700/87
} 
Short-crested waves occur in many situations, one of the most important being reflection of incoming waves off a seawall, jetty or depth discontinuity. Other situations in which short-crested waves occur include diffraction behind an object such as an island or man-made breakwater, or the arrival of swell from two different storm centres.

The short-crested wave problem has been studied using perturbation expansions by various authors. Fuchs [8] obtained a second order solution and Chappelear [3] calculated a third order solution. Both solutions were in dimensional form using an expansion parameter related to the ratio of the waveheight to the wavelength parallel to the wall. This does not allow the standing wave limit to be calculated. Hsu, Tsuchiya and Silvester [10] calculated a third order solution in non-dimensional form using an expansion parameter related to the ratio of the waveheight to the wavelength of the incident wave, thus allowing them to calculate the standing wave limit.

Roberts [16] investigated the infinite depth case in great detail. He computed solutions via a perturbation expansion of 27 th order in wave steepness. He found that the phenomenon of harmonic resonance occurs at some angles causing the magnitude of some higher order coefficients to increase rapidly and concluded that the perturbation series has an everywhere zero radius of convergence. By using Padé approximants he was able to obtain convergent solutions for the frequency, potential energy and kinetic energy nearly up to the maximum wave steepness which was estimated from the Padé approximants. Numerical schemes have also been used by Bryant [2] and Roberts and Schwartz [15]. Bryant considered the two-dimensional Korteweg-de Vries equation which models two progressive wavetrains propagating obliquely to each other in shallow water. His numerical scheme is accurate to $O(a / d)$ where $a$ is wave amplitude and $d$ is the water depth. Roberts and Schwartz's method is for arbitrary depth and they present results for deep water.

The standing wave problem has been investigated to third order by Tadjbakhsh and Keller [19] and to fourth order by Goda [9]. Goda calculated the wave pressures acting on the wall due to fully reflected waves and obtained good agreement with experiment. Fenton [7] examined the short-crested wave problem to third order and obtained expressions valid in deeper water for the depth integrated wave force and moment exerted on the wall. One of his main findings was the non-intuitive result that the maximum force per unit length is caused by obliquely incident waves rather than standing waves.

The aim of this work is to generalize the results of Roberts [16] to arbitrary depth. In Section 2 the mathematical formulation of the problem is given and in Section 3 a procedure is described for the calculation of the coefficients of a perturbation expansion to general order $N$. Coefficients are computed up to $N=35$. The phenomenon of harmonic resonance is discussed in Section 4. In 
Section 5 Padé approximants are used to calculate wave properties such as maximum waveheight, frequency and energies although accurate estimates are difficult to obtain for shallow water. The force exerted by waves on a seawall is an important design criterion. In Section 6 the force exerted on the wall by fully reflected waves striking the wall at arbitrary angle and depth is calculated, hence extending the results of Fenton [7] to shallow water. Our results for the maximum depth-integrated onshore force are compared with experiment in the standing-wave limit. Results are presented for the maximum depth-integrated onshore and offshore forces.

\section{System of equations}

For convenience the same variables and notation are used as by Roberts [16] and Hsu et al [10]. As shown in Figure 1, the $x$-axis is along the reflecting wall, the $z$-axis is perpendicular to the wall and the $y$-axis is vertically up. We assume two successive wavefronts (represented by the dashed lines) separated by the wavelength $L$ strike the wall at an angle $\theta$ to the $z$-axis and are perfectly reflected. Then the wavelength in the $x$-direction $L_{x}$ is $L / \sin \theta$ and the wavelength in the $z$-direction $L_{z}$ is $L / \cos \theta$. In the limit $\theta \rightarrow 0^{\circ}$ we get the two-dimensional standing wave and in the limit $\theta \rightarrow 90^{\circ}$ we get the two-dimensional progressive wave. We assume the fluid is incompressible and inviscid and the fluid motion is irrotational. Thus we look for a velocity potential $\phi(x, y, z, t)$
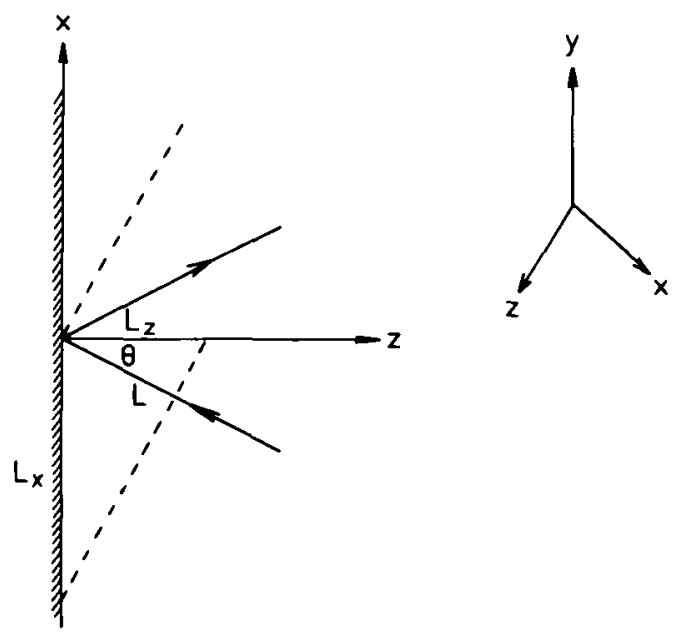

Figure 1. Definition sketch of the short-crested wave system. 
such that

$$
\nabla^{2} \phi=0, \quad y<\eta(x, z, t)
$$

where $\eta$ is the surface elevation.

We shall non-dimensionalize the variables and equations with respect to the reference length $1 / k$ (where $k$ is the wave number $2 \pi / L$ ) and the reference time $(g k)^{-1 / 2}$. The non-dimensional wave numbers in the $x$ and $z$ directioñ are $p$ and $q$ respectively where

$$
p=\sin \theta, \quad q=\cos \theta
$$

Therefore we define

$$
X=p x-\omega t, \quad Z=q z,
$$

and look for $\phi(X, y, Z)$ and $\eta(X, Z)$, periodic in both $X$ and $Z$ with period $2 \pi$. The full problem in the new non-dimensional co-ordinates is then

$$
p^{2} \phi_{X X}+\phi_{y y}+q^{2} \phi_{Z Z}=0, \quad y<\eta(X, Z),
$$

with the kinematic boundary condition, which means that no fluid crosses the free surface,

$$
-\omega \eta_{X}+p^{2} U \eta_{X}-V+q^{2} W \eta_{Z}=0
$$

and the constant pressure condition which, via Bernoulli's equation, is

$$
-\omega U+\eta+\frac{1}{2}\left(p^{2} U^{2}+V^{2}+q^{2} W^{2}\right)=C,
$$

where $C$ is a constant chosen so that the mean water level is $y=0 . p U, V$ and $q W$ are the $x, y$ and $z$ fluid velocity components respectively at the surface $y=\eta(X, Z)$, that is

$$
\begin{gathered}
U(X, Z)=\phi_{X}(X, \eta, Z), \\
V(X, Z)=\phi_{y}(X, \eta, Z), \\
W(X, Z)=\phi_{Z}(X, \eta, Z) .
\end{gathered}
$$

Also there is the condition of zero vertical velocity on the fluid bed

$$
\phi_{y}=0 \text { on } y=-d \text {. }
$$

\section{The perturbation expansion}

Non-linear problems rarely yield exact solutions. To obtain solutions for this problem a perturbation procedure is used. The perturbation parameter we use is a non-dimensional waveheight $h$ defined by

$$
h=\frac{1}{2}[\eta(0,0)-\eta(\pi, 0)],
$$

where $\eta(0,0)$ and $\eta(\pi, 0)$ are the peak and trough of the wave respectively. 
We expand the quantities $\phi, \eta, \omega, C, U, V$ and $W$ as power series in the parameter $h$

$$
\begin{aligned}
& \phi=\sum_{r=1}^{\infty} h^{r} \phi_{r}, \quad \eta=\sum_{r=1}^{\infty} h^{r} \eta_{r}, \quad \omega=\sum_{r=1}^{\infty} h^{r} \omega_{r}, \quad C=\sum_{r=1}^{\infty} h^{r} C_{r}, \\
& U=\sum_{r=1}^{\infty} h^{r} U_{r}, \quad V=\sum_{r=1}^{\infty} h^{r} V_{r}, \quad W=\sum_{r=1}^{\infty} h^{r} W_{r} .
\end{aligned}
$$

Substituting the above power series into the set of equations (2.4)-(2.8) we obtain the following set of equations at each order in $h$, which shall be solved successively:

$$
\left.\begin{array}{r}
p^{2} \phi_{r X X}+\phi_{r y y}+q^{2} \phi_{r Z Z}=0 \\
\phi_{r y}=0 \text { on } y=-d \\
\omega_{0} \eta_{r}+V_{r}=A_{r} \\
-\omega_{0} U_{r}+\eta_{r}=B_{r}+C_{r}
\end{array}\right\} \quad r=1,2,3, \ldots
$$

where

$$
\begin{aligned}
A_{r} & =\sum_{s=1}^{r-1}\left[-\omega_{r-s} \eta_{s X}+p^{2} U_{r-s} \eta_{s X}+q^{2} \omega_{r-s} \eta_{s Z}\right], \\
B_{r} & =\sum_{s=1}^{r-1}\left[\omega_{r-s} U_{s}-\frac{1}{2}\left(p^{2} U_{r-s} U_{s}+V_{r-s} V_{s}+q^{2} W_{r-s} W_{s}\right)\right] .
\end{aligned}
$$

We consider first the leading order linear problem

$$
\begin{gathered}
p^{2} \phi_{1 X X}+\phi_{1 y y}+q^{2} \phi_{1 Z Z}=0, \\
\dot{\phi}_{1 y}=0 \text { on } y=-d, \\
\omega_{0} \eta_{1 X}+\phi_{1 y}=0 \text { on } y=0, \\
-\omega_{0} \phi_{1 x}+\eta_{1}=0 \text { on } y=0,
\end{gathered}
$$

and try for a solution of the form

$$
\begin{aligned}
& \eta=\cos (X) \cos (Z), \\
& \phi=\sin (X) \cos (Z) f(y) .
\end{aligned}
$$

Laplace's equation and the fluid bed condition require $f(y)$ to be of the form

$$
f(y)=A \cosh (y+d),
$$

while the two free surface boundary conditions result in

$$
\omega_{0}^{2}=\tanh (d) \text { and } A=\frac{1}{\omega_{0} \cosh (d)} .
$$


Therefore the first order solution can be written as

$$
\begin{aligned}
\eta_{1} & =\cos (X) \cos (Z), \\
\phi_{1} & =\frac{1}{\omega_{0}} \sin (X) \cos (Z) \frac{\cosh (y+d)}{\cosh (d)}, \\
\omega_{0}^{2} & =\tanh (d) .
\end{aligned}
$$

The form of this first order solution results in the $r$ th order solution having the form

$$
\begin{aligned}
\phi_{r} & =\sum_{m, n} b_{r m n} \sin (m X) \cos (n Z) \frac{\cosh \left[\alpha_{m n}(y+d)\right]}{\cosh \left(\alpha_{m n} d\right)}, \\
\eta_{r} & =\sum_{m, n} a_{r m n} \cos (m X) \cos (n Z),
\end{aligned}
$$

where $\alpha_{m n}^{2}=(p m)^{2}+(q n)^{2}$. A further restriction on the solution is the triangular symmetry of the wave

$$
\eta(X, Z)=\eta(Z, X)
$$

which restricts $m$ and $n$ to be both even or both odd.

A complication arises in the calculation of $U_{r}, V_{r}$ and $W_{r}$ as they are the derivatives of $\phi_{r}$ evaluated on the free surface $y=\eta(X, Z)$, which is itself expressed as a series in $h$. An efficient method of computing them, outlined in Roberts [16], is to expand $\exp [\alpha \eta(X, Z)]$ in a Taylor series in $h$. Let

$$
\exp [\alpha \eta(X, Z)]=\sum_{r=0}^{\infty} h^{\prime} E_{r}(X, Z, \alpha)
$$

then

$$
E_{0}=1, \quad E_{r}=\frac{\alpha}{r} \sum_{s=1}^{r} s \eta_{s} E_{r-s}, \quad r=1,2,3, \ldots,
$$

and upon defining

$$
\begin{aligned}
& G_{r}(X, Z, \alpha)=\frac{\exp (\alpha d)}{\cosh (\alpha d)} E_{r}(X, Z, \alpha)+\frac{\exp (-\alpha d)}{\cosh (\alpha d)} E_{r}(X, Z,-\alpha), \\
& G_{r}^{\prime}(X, Z, \alpha)=\frac{\exp (\alpha d)}{\cosh (\alpha d)} E_{r}(X, Z, \alpha)-\frac{\exp (-\alpha d)}{\cosh (\alpha d)} E_{r}(X, Z,-\alpha),
\end{aligned}
$$

then

$$
\begin{gathered}
U_{r}=\phi_{r X}(X, 0, Z)+\bar{U}_{r}, \\
V_{r}=\phi_{r y}(X, 0, Z)+\bar{V}_{r}, \\
W_{r}=\phi_{r Z}(X, 0, Z)+\bar{W}_{r},
\end{gathered}
$$


where

$$
\begin{aligned}
& \bar{U}_{r}=\sum_{m, n} \sum_{s=1}^{r} m b_{s m n} \cos (m X) \cos (n Z) G_{r-s}\left(X, Z, \alpha_{m n}\right), \\
& \bar{V}_{r}=\sum_{m, n} \sum_{s=1}^{r} \alpha_{m n} b_{s m n} \sin (m X) \cos (n Z) G_{r-s}^{\prime}\left(X, Z, \alpha_{m n}\right), \\
& \bar{W}_{r}=-\sum_{m, n} \sum_{s=1}^{r} n b_{s m n} \sin (m X) \sin (n Z) G_{r-s}\left(X, Z, \alpha_{m n}\right) .
\end{aligned}
$$

Substituting (3.14) into equations (3.3) gives

$$
\omega_{0}^{2} \phi_{r X X}+\phi_{r y}=A_{r}-\bar{V}_{r}-\omega_{0} B_{r X}-\omega_{0}^{2} \bar{U}_{r X} \quad \text { on } y=0
$$

Equation (3.16) is used to solve for the coefficients $b_{r m n}$. For odd $r$, a term of the form $\sin (X) \cos (Z) \cosh (y+d) / \cosh (d)$ will be found on the right hand side of (3.16). Now the fundamental $\sin (X) \cos (Z) \cosh (y+d) / \cosh (d)$ is a solution of the associated homogeneous differential equation, forcing unallowable secular terms into the solution. Since the coefficient of this term is of the form $\left(a+2 \omega_{r-1}\right)$ where $\omega_{r-1}$ is unknown, $\omega_{r-1}$ can be set to eliminate this secular term. Then $\eta_{r}$ can be found from the last of equations (3.3).

A program to do these calculations was written in FORTRAN and uses computer time like $N^{7}$ and store like $N^{4}$ where $N$ is the highest order calculated. The program takes 75 minutes to calculate double precision coefficients up to order $N=35$ on a VAX $11 / 780$. The expansions obtained agree in the infinite depth limit with Roberts' [16] coefficients and also agree with Fenton's [7] third order coefficients over a range of depths. A more indirect confirmation of accuracy can be seen later in Section 6 when results for the maximum depth-integrated onshore wave force are compared with Goda's [9] experimental values.

\section{Harmonic resonance}

For various angles $\theta$, depending on the depth $d$, a division by zero occurs in the calculation of some coefficients at particular orders. In the region near these particular angles, division by a small number causes these coefficients to increase rapidly with the order. This phenomemon is indicative of the occurrence of harmonic resonance. 
The equation which relates the depth $d$ and the angle $\theta$ for which harmonic resonance occurs with respect to the $(m, n)$ th harmonic, namely $\sin (m X) \cos (n Z) \cosh \left[\alpha_{m n}(y+d)\right] / \cosh \left(\alpha_{m n} d\right)$, is

$$
\alpha_{m n} \tanh \left(\alpha_{m n} d\right)=m^{2} \tanh (d) .
$$

These divisions by zero occur because the $(m, n)$ th harmonic is a homogeneous solution of the iinear differential equation and at some order it occurs in the right hand side of (3.16). Physically, the fundamental $\sin (X) \cos (Z) \cosh (y+$ $d) / \cosh (d)$ excites the harmonic which travels at the same phase speed. Over a long time scale the resonance allows continual redistribution of energy between the fundamental and the resonant harmonic.

Table 1 shows the angles at which harmonic resonance occurs with respect to the $(m, n)$ th harmonic at depth $d=0.8$ for $N$ up to 35 . If this table is compared

TABLE 1. Angles at which harmonic resonance occurs for $d=0.8$ up to $N=35$

\begin{tabular}{|c|c|c|c|c|c|c|c|}
\hline$n$ & $m=1$ & $m=2$ & $m=3$ & $m=4$ & $m=5$ & $m=6$ & $m=7$ \\
\hline 3 & $90.00^{\circ}$ & & & & & & \\
\hline 4 & & $57.71^{\circ}$ & & & & & \\
\hline 5 & $90.00^{\circ}$ & & & & & & \\
\hline 6 & & $70.90^{\circ}$ & & & & & \\
\hline 7 & $90.00^{\circ}$ & & $35.17^{\circ}$ & & & & \\
\hline 8 & & $76.18^{\circ}$ & & & & & \\
\hline 9 & $90.00^{\circ}$ & & $52.47^{\circ}$ & & & & \\
\hline 10 & & $79.12^{\circ}$ & & & & & \\
\hline 11 & $90.00^{\circ}$ & & $60.76^{\circ}$ & & & & \\
\hline 12 & & $81.01^{\circ}$ & & $29.55^{\circ}$ & & & \\
\hline 13 & $90.00^{\circ}$ & & $65.88^{\circ}$ & & & & \\
\hline 14 & & $82.33^{\circ}$ & & $42.81^{\circ}$ & & & \\
\hline 15 & $90.00^{\circ}$ & & $69.41^{\circ}$ & & & & \\
\hline 16 & & $83.31^{\circ}$ & & $50.56^{\circ}$ & & & \\
\hline 17 & $90.00^{\circ}$ & & $72.01^{\circ}$ & & $13.03^{\circ}$ & & \\
\hline 18 & & $84.01^{\circ}$ & & $55.89^{\circ}$ & & & \\
\hline 19 & $90.00^{\circ}$ & & $74.01^{\circ}$ & & $30.28^{\circ}$ & & \\
\hline 20 & & $84.67^{\circ}$ & & $59.85^{\circ}$ & & & \\
\hline 21 & $90.00^{\circ}$ & & $75.60^{\circ}$ & & $39.10^{\circ}$ & & \\
\hline 22 & & $85.16^{\circ}$ & & $62.94^{\circ}$ & & & \\
\hline 23 & $90.00^{\circ}$ & & $76.90^{\circ}$ & & $45.16^{\circ}$ & & \\
\hline 24 & & $85.57^{\circ}$ & & $65.43^{\circ}$ & & $5.26^{\circ}$ & \\
\hline 25 & $90.00^{\circ}$ & & $77.98^{\circ}$ & & $49.74^{\circ}$ & & \\
\hline 26 & & $85.91^{\circ}$ & & $67.48^{\circ}$ & & $23.84^{\circ}$ & \\
\hline 27 & $90.00^{\circ}$ & & $78.90^{\circ}$ & & $53.38^{\circ}$ & & \\
\hline 28 & & $86.21^{\circ}$ & & $69.20^{\circ}$ & & $32.22^{\circ}$ & \\
\hline 29 & $90.00^{\circ}$ & & $79.68^{\circ}$ & & $56.35^{\circ}$ & & \\
\hline 30 & & $86.46^{\circ}$ & & $70.67^{\circ}$ & & $38.08^{\circ}$ & \\
\hline 31 & $90.00^{\circ}$ & & $80.36^{\circ}$ & & $58.85^{\circ}$ & & \\
\hline 32 & & $86.68^{\circ}$ & & $71.94^{\circ}$ & & $42.60^{\circ}$ & \\
\hline 33 & $90.00^{\circ}$ & & $80.95^{\circ}$ & & $60.97^{\circ}$ & & $9.83^{\circ}$ \\
\hline 34 & & $86.88^{\circ}$ & & $73.06^{\circ}$ & & $42.26^{\circ}$ & \\
\hline 35 & $90.00^{\circ}$ & & $81.48^{\circ}$ & & $62.81^{\circ}$ & & $22.09^{\circ}$ \\
\hline
\end{tabular}


to Tables 1 and 2 from Roberts [16] it is seen that at all depths the common features of the distribution of harmonic resonances are: 1) an infinite number of resonances at $\theta=90^{\circ}$ (for $m=1$ ); and 2) as $N$ is increased the number of resonances increases, in fact as $N \rightarrow \infty$ a zero-divisor will occur arbitrarily near every angle between $\theta=0^{\circ}$ and $\theta=90^{\circ}$. Therefore the perturbation series has an everywhere zero radius of convergence.

Table 2 shows the depths at which harmonic resonance occurs for standing waves, $\theta=0^{\circ}$, with respect to the $(m, n)$ th harmonic. Due to the non-dispersive nature of shallow water waves the number of resonances increases swiftly as the depth decreases. The information contained in the perturbation expansion from a point close to such a zero-divisor will be degraded due to the use of finite precision arithmetic. So for shallow depths (say $d \leqslant 0.5$ ) accurate estimates of wave properties such as maximum waveheight are difficult to obtain.

We wish to use the perturbation series to calculate such quantities as maximum waveheight, frequency, kinetic energy, potential energy and pressure on the wall. The series for a quantity $f(h)$ will have the form

$$
f(h)=a_{0}+a_{1} h+a_{2} h^{2}+\cdots+a_{n+m} h^{n+m},
$$

where $n+m=N$, the highest order calculated. Now $f(h)$ will converge only for $|h|<\left|h^{*}\right|$ where $h^{*}$ is the location of the singularity nearest the origin. But if $h^{*}$ corresponds to a weak singularity (which is typical if it is due to harmonic resonance) then the series will be affected by stronger more distant singularities. The $[m / n]$ Padé approximant of the series $f(h)$ is

$$
[m / n]=\frac{b_{0}+b_{1} h+b_{2} h^{2}+\cdots+b_{m} h^{m}}{c_{0}+c_{1} h+c_{2} h^{2}+\cdots+c_{n} h^{n}}
$$

where the $b_{i}$ and $c_{i}$ are chosen so that the Maclaurin series of $[\mathrm{m} / \mathrm{n}]$ is the same as that of $f(h)$ to order $m+n$. The Padé approximant places a simple pole (and a nearby corresponding zero) at the singularities of $f(h)$ which are due to harmonic resonance. They will often converge to a solution of the original equations for real $h$ past $h^{*}$.

Figure 2 shows the locations of pole singularities due to harmonic resonance at $2^{\circ}$ intervals for $d=0.8$ which appear consistently in the [17/18], [17/17], [16/17] and [16/16] Padé approximants of the crest-curvature (the expansion of $\eta_{X X}(0,0)$ ). As mentioned in Roberts [16] and Chen and Saffman [4] near these pole singularities, due to harmonic resonance, in general three distinct solutions exist (see Roberts [13]). So by crossing the lines of Figure 2 the derived solution will jump from one branch of the solution to a different solution branch. However, the "distance" between the different solution branches is typically very small, being of the order of $h^{\max (m, n) / 3}$ when the jump is due to harmonic resonance with the $(m, n)$ th harmonic (see Roberts [13]). 


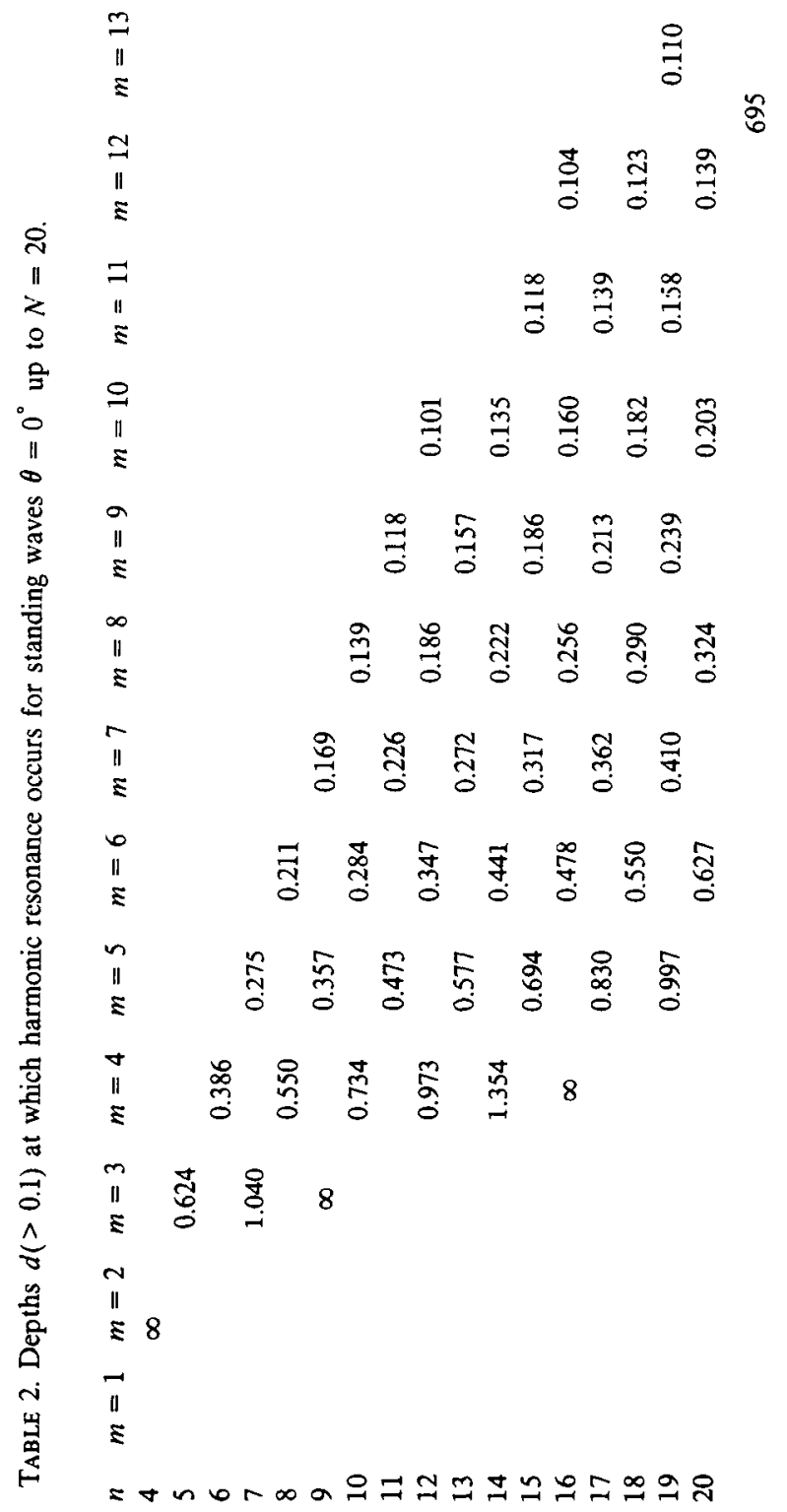




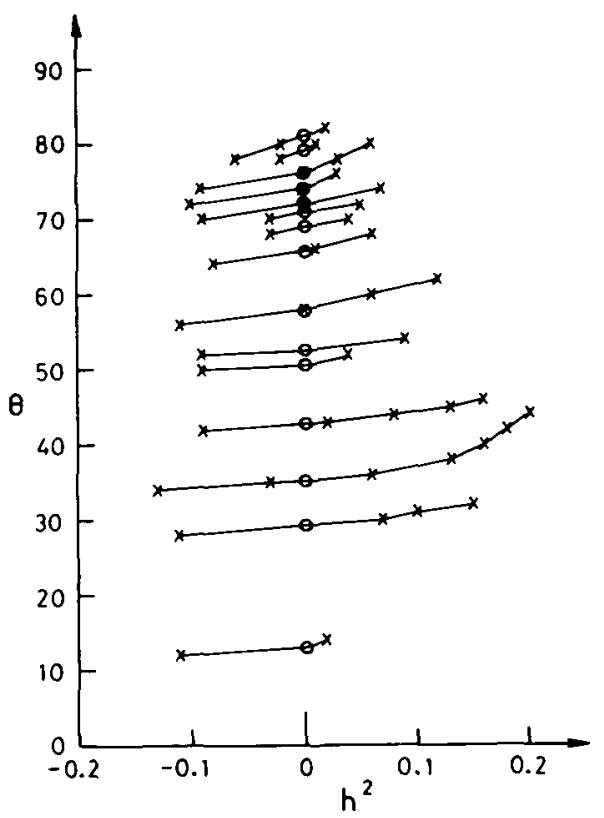

Figure 2. Locations of pole singularities due to harmonic resonance $(X)$ at $2^{\circ}$ intervals for $d=0.8$. Also plotted $\left({ }^{\circ}\right)$ are the angles at which zero-divisors occur (the straight lines are for visualisation purposes only).

The physical relevance of these steady solutions near harmonic resonance is determined by the behaviour in time of solutions to the original equations. Roberts [16] suggested that the behaviour of these solutions is likely to be qualitatively similar to the results of McGoldrick [11], [12]. McGoldrick studied the behaviour of unsteady solutions near second and third resonance using the method of multiple scales. He found that the fundamental and resonant harmonic interact over a long timescale. Our problem is of a slightly different nature though, and so more general interactions need to be studied before any conclusions can be reached.

\section{Results}

\subsection{Maximum waveheight estimates}

As well as information about the pole singularities due to harmonic resonance (which are matched by nearby zeros and hence are weak) the perturbation series contains information about singularities resulting from physical limitations on the waveheight. Hence the maximum waveheight can be estimated by calculating the $[m / m]$ and $[m / m+1]$ Padé approximants via continued fractions (see Bender and Orszag [1]). These operations were applied to the series of $\eta_{X X}(0,0)$ which is 
the curvature in the $x$-direction at the wave's peak ( $h$ positive) or the wave's trough ( $h$ negative). The singularity which limits the waveheight is expected to be due to a sharp crest at the peak of a wave of maximum waveheight which would correspond to infinite curvature.

When inspecting the real poles and zeros of the Padé approximants we typically find: 1) pole-zero pairs with small separation $\left(\leqslant 10^{-3}\right)$ which are due to harmonic resonancc; and 2) unmmatched poie singuiarities of which the smallest positive one is taken to be an estimate of the maximum waveheight. To be considered, these poles and zeros must be consistent from one Padé approximant to the next and not just a quirk of any particular approximant.

The maximum wave height estimates are taken from the location of the poles of [17/18], [17/17], [16/17] and [16/16] Padé approximants. The closest group of three of these poles were taken to measure the waveheight and give an estimate of its error. In Figure 3 we show the maximum waveheight estimates for $d=0.8$ at $2^{\circ}$ intervals. The maximum waveheight estimate for the progressive wave $\left(\theta=90^{\circ}\right)$ is taken from Cokelet [5] (the crosses in Figure 3 represent the $e^{-d}=0.5$ and $e^{-d}=0.4$ maximum waveheight estimates in Cokelet's Table 3 ). In general we obtain estimates with small error bars and which are consistent as $\theta$ is varied. Some difficulties were encountered in the region $\left[0^{\circ}, 30^{\circ}\right]$ where complex conjugate pairs of poles which lay near the real axis were used to obtain estimates of

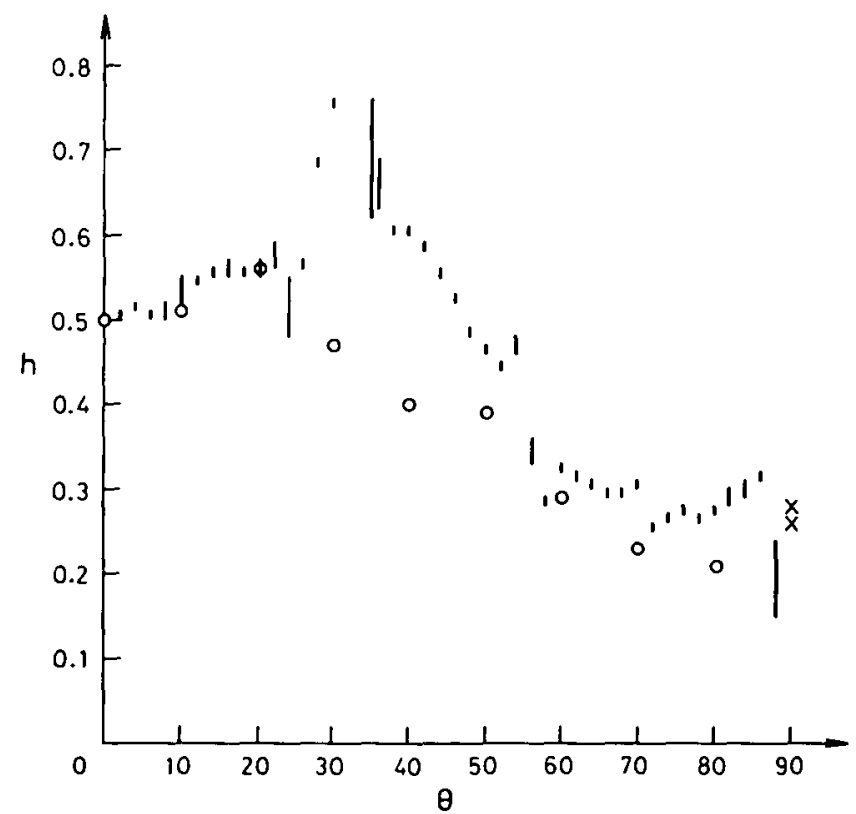

Figure 3. Estimates of maximum waveheights (with error bars) at $2^{\circ}$ intervals for $d=0.8$. Also shown is the maximum waveheight for the progressive wave $(x)$ from Cokelet (1977), and the height to which frequencies are drawn in Figure $4(\circ)$. 
the maximum waveheights. Estimates remain uncertain in the region $\left[30^{\circ}, 35^{\circ}\right]$ as the information is degraded due to the proximity of the strong $(3,7)$ and $(4,12)$ harmonic resonances. Also the graph shows a sudden drop in maximum waveheight in the regions $\theta=35^{\circ}$ to $\theta=38^{\circ}$ and $\theta=54^{\circ}$ to $\theta=58^{\circ}$. These drops in maximum waveheight are associated with the obtained solution being on a different branch of solutions on crossing from one side to the other of the singularities associated with the $(3,7)$ and $(2,4)$ resonances respectively. Similarly the smaller jump in maximum waveheight in the region $\theta=70^{\circ}$ to $\theta=72^{\circ}$ is associated with the $(2,6)$ resonance.

\subsection{Estimates of frequency}

To plot the frequency, a 17 term series in $h^{2}$ from the 35 th order expansion was used. The average of the four [8/9], [8/8], [7/8] and [7/7] Padé approximants was taken, the worst result removed and then the average of the remaining three results was plotted. An estimate of the error was taken as the sum of the absolute deviations of the three results from their mean. When this estimated error reached $4 \times 10^{-5}$ plotting was stopped. This waveheight which is shown on Figure 3 usually represented a height of between $80 \%$ to $100 \%$ of the estimated maximum waveheight, although between $\theta=30^{\circ}$ and $\theta=40^{\circ}$ plotting was stopped lower than this.

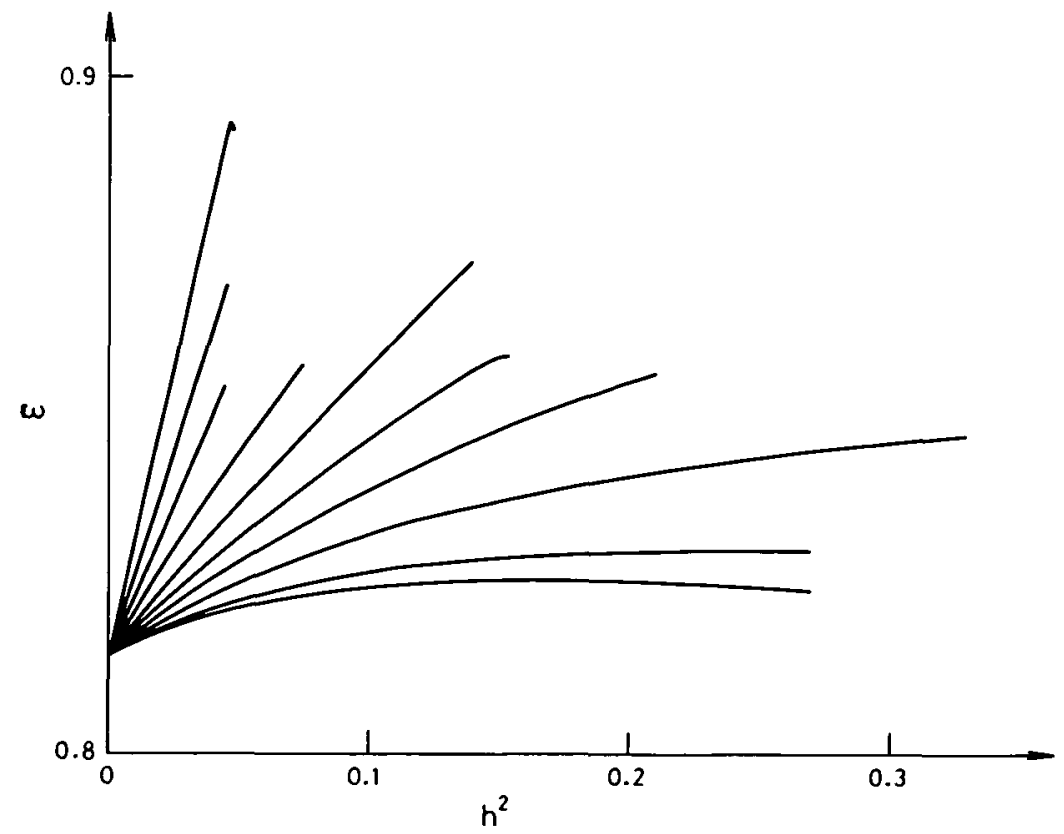

Figure 4. Frequency of a short-crested wave as a function of waveheight squared for fixed $\theta$ at $d=0.8$. The frequencies are plotted from $\theta=0^{\circ}$ to $\theta=90^{\circ}$ at $10^{\circ}$ intervals in a counter-clockwise direction. 
The frequencies from $\theta=0^{\circ}$ to $\theta=90^{\circ}$ at $10^{\circ}$ intervals at depth $d=0.8$ are drawn in Figure 4. The $\theta=90^{\circ}$ (the progressive wave limit) curve is taken from Cokelet [5] (obtained by a linear interpolation of the $e^{-d}=0.5$ and $e^{-d}=0.4$ frequency curves in Cokelet's Figure 5). For $\theta \leqslant 10^{\circ}$ the frequency increased with waveheight to a maximum below the maximum waveheight (in deeper water, $d \geqslant 1.05$, this behavior is reversed and the frequency decreases with waveheight to a minimum below the maximum waveheight). In the rest of the parameter range $\theta>10^{\circ}$, frequency was an increasing function of waveheight with no definite evidence being found of a frequency maximum.

\subsection{The free surface}

In Figure 5, free surfaces are drawn for various $\theta$ at depth $d=0.8$. At small $\theta$ (near the standing wave limit) the crest is sharply peaked in the $z$-direction but well-rounded in the $x$-direction. In the middle range the wave exhibits the typical characteristics of short-crested waves, namely a symmetric pattern of short steep crests and flat troughs. As $\theta \rightarrow 90^{\circ}$ a pattern of long (but not infinite) flat peaks and flat troughs appears; the so-called long-crested wave.

\section{Wave forces on a vertical wall}

The force exerted by waves being reflected from a seawall is an important design criterion. The maximum force exerted by waves being reflected from a seawall can be found by varying the parameters associated with this problem. The

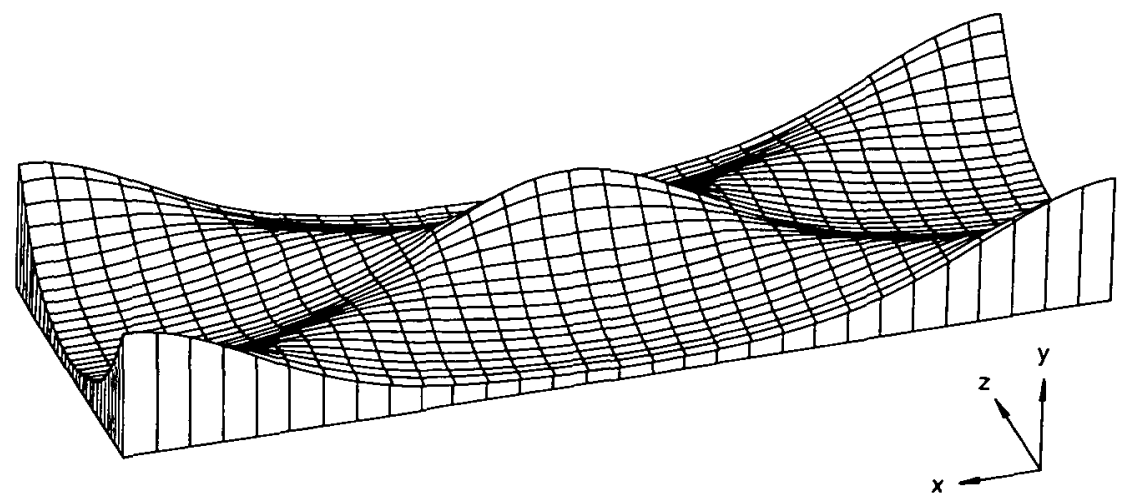

Figure 5. Perspective drawings of a one wavelength rectangle of the short-crested wave's free-surface for $d=0.8$ and various $\theta$ and $h$.
(a) $\theta=10^{\circ}, h=0.4$
(b) $\theta=40^{\circ}, h=0.6$
(c) $\theta=80^{\circ}, h=0.27$ 

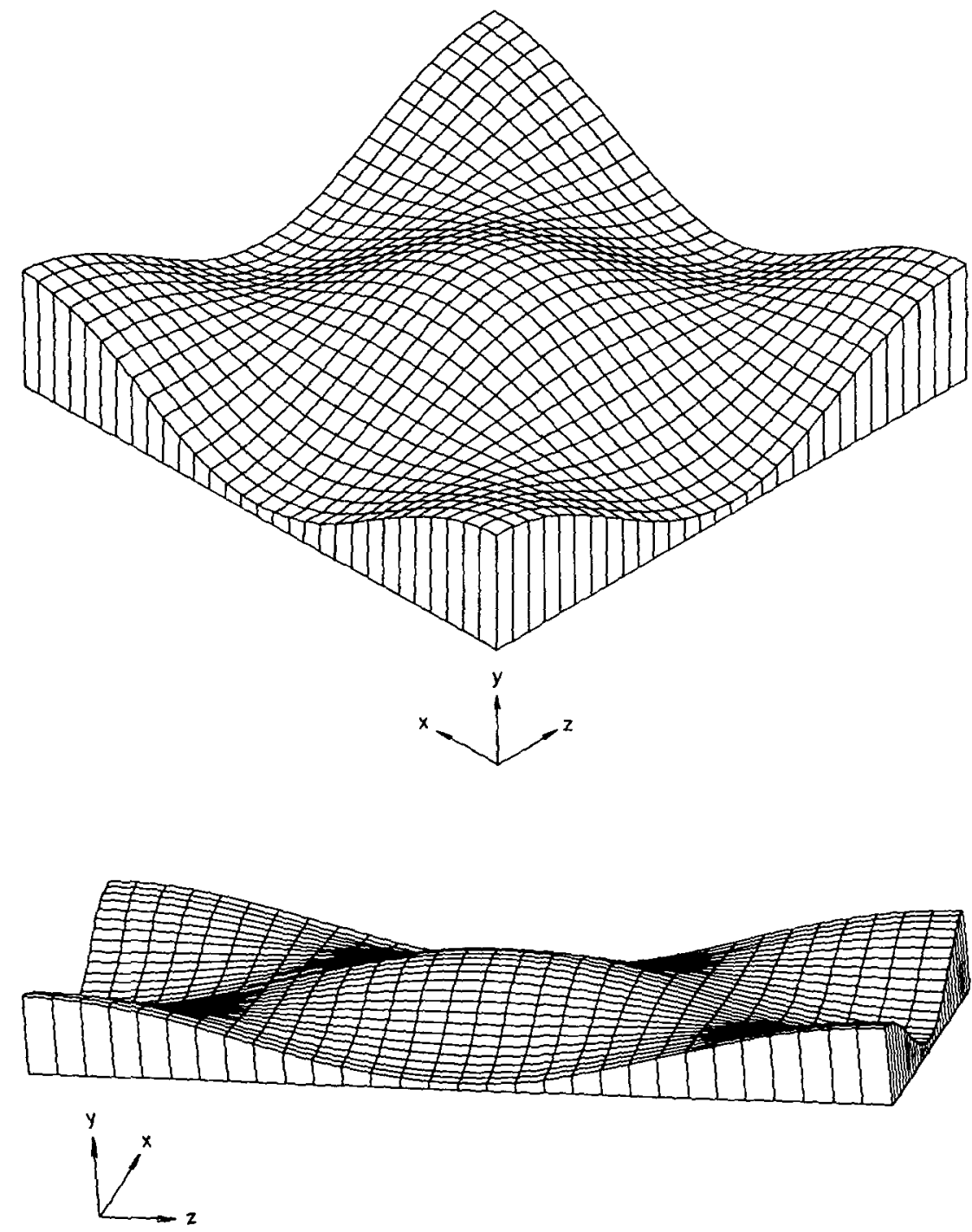

Figure 5 (continued).

parameters are the waveheight $(h)$, the water depth $(d)$, the angle of incidence $(\theta)$, the wave number $(k)$ and the phase of the wave motion $(\chi)$. Goda [9] investigated the standing wave problem (when $\theta=0^{\circ}$ ) to fourth order. Goda used the usual perturbation expansion of $\phi, \eta$ and $\omega$ in a perturbation parameter related to waveheight. He calculated the wall pressure using Bernoulli's equation 
and drew graphs of the pressure at the mean water level, at the water bottom and of the depth integrated maximum onshore wave force. He then compared these results with experiment and obtained good agreement. Fenton [7] examined the short-crested wave problem to third order and obtained expressions for the depth integrated wave force on the wall and the depth integrated wave moment about the base of the wall. He compared his results in the standing wave limit with Goda's experimental results and achieved a good fit only for deeper water $(d \geqslant 1.2)$. The reason for this is that as the water becomes shallower the higher-order terms in the perturbation expansion grow in size (relative to the lower-order terms) and hence become increasingly important.

The main limitation of previous work is that the wave force is not calculated in the situation which subjects the wall to the greatest onshore wave force, i.e., the obliquely incident wave in shallow water. The standing wave work of Goda is valid in shallow water but only for normal approach to the wall $\left(\theta=0^{\circ}\right)$ while the short-crested work of Fenton is valid only for $d>1.2$. The present work calculates the wall force caused by a short-crested wave system, valid for waves in all but the shallowest depths and at all angles of incidence.

\subsection{Derivation of equations}

The situation is as described previously with a wall at $z=0$. The depth-integrated force per unit length of the wall is given by

$$
F(x, t)=\int_{-d}^{\eta(x, 0, t)} p(x, y, 0, t) d y,
$$

where Bernoulli's theorem gives

$$
p=-\rho g y-\rho \phi_{t}-\frac{1}{2} \rho|\nabla \phi|^{2}+C^{\prime},
$$

where $C^{\prime}$ is a constant added so that the mean water level is held at $y=0$. Nondimensionalising the equation and integrating gives the non-dimensional force $F^{\prime}=k^{2} F / \rho g$, where

$$
F^{\prime}=\frac{1}{2} d^{2}-\frac{1}{2} \eta^{2}+\int_{-d}^{\eta(X, 0)}\left(\omega \phi_{X}-\frac{1}{2}|\nabla \phi|^{2}+C\right) d y \quad(Z=0) .
$$

The term $\frac{1}{2} d^{2}$ gives the hydrostatic force that the wall is subjected to by an undisturbed region of water. Let $F^{\prime \prime}=F^{\prime}-\frac{1}{2} d^{2}$ be the force due to the waves. Then

$$
F^{\prime \prime}=\frac{1}{2} \eta^{2}+\int_{-d}^{\eta(X, 0)}\left(\omega \phi_{X}-\frac{1}{2}|\nabla \phi|^{2}+C\right) d y \quad(Z=0) .
$$

By substituting the series for $\eta$ and $\phi$ into the above integral, the coefficients of the series for $F^{\prime \prime}$ can be calculated. The resultant expansion is of the form

$$
F^{\prime \prime}=\sum_{r=1}^{\infty} h^{r} F_{r}
$$


where

$$
F_{r}=\sum_{m=0}^{r} F_{r m} \cos (m x)
$$

The calculations for the coefficients of the series (6.5) were programmed in FORTRAN and the results check against Fenton's third order results for the tabulated case $d=2, \theta=36.87^{\circ}$ ).

\subsection{Comparision with the standing wave limit}

The theory can be compared in the standing wave limit to Goda's results. He developed a fourth-order theory and also performed experiments to check his results. Here we compare this theory with the results given in Figure 6 of Goda's paper, which is a graph of the maximum depth-integrated onshore force $(P / \rho g H d)$ versus wave steepness $(H / L)$ where $H$ is half the crest to trough waveheight and $L$ is the wavelength.

Harmonic resonance occurs with respect to the $(m, n)$ th harmonic for combinations of depth, $d$, and angle, $\theta$, given by (4.1). In the standing wave limit $\theta \rightarrow 0$ we get $\alpha_{m n} \rightarrow n$ so the equation becomes

$$
n \frac{\tanh (n d)}{\tanh (d)}=m^{2}
$$

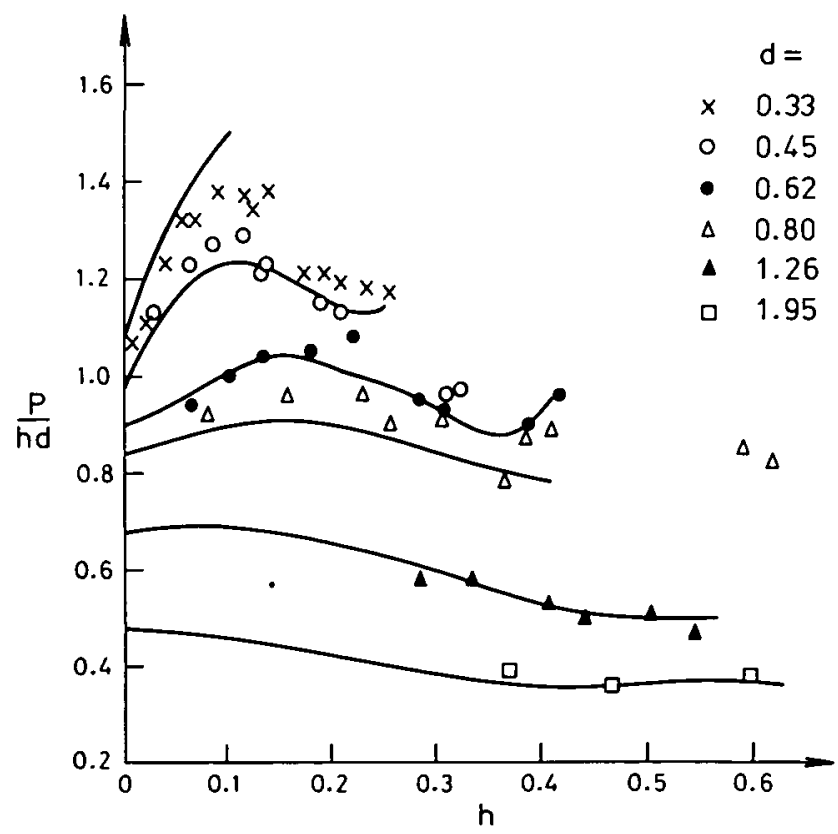

Figure 6. Maximum onshore depth integrated force per unit length versus waveheight in the standing wave limit. Shown is a comparison between present theory (lines) and the experimental work of Goda (symbols). 
which is the uniqueness condition obtained by Concus [6]. Table 2 gives the depths at which harmonic resonance occurs with respect to the $(m, n)$ th harmonic in the standing wave limit. Depths below $d=0.1$ have been excluded from the table as they are well outside the region of interest $(d \geqslant 0.3)$. To obtain convergence using Padé approximants, ten to twenty terms in the series were generally used, more terms being required in shallow water than in deep water.

In order to make the comparison the non-dimensional waveheight, $h$ and the nondimensional water depth, $d$ must be related to Goda's variables. The necessary conversions are

$$
h=2 \pi(H / L), \quad d=2 \pi(D / L) .
$$

$P / h d$ versus $h$ is plotted in Figure 6 for Goda's chosen depths of $d=1.95,1.26$, $0.80,0.62,0.45$ and 0.33 . As can be seen from Figure 6 an excellent comparison is obtained with the experimental results of Goda. One point of disagreement is for $d=0.33$. Goda obtained experimental results which were much higher than it was possible to obtain solutions. One explanation for this is that the steep standing waves in shallow water measured by Goda may not have been precisely periodic. Another possible explanation is that, for standing waves, many harmonic resonances occur near $d=0.33$ (see Table 2) and these are degrading the information contained in the perturbation series.

\subsection{The maximum load}

The variables which must be varied when searching for the situation which results in the maximum load on the wall are 1) phase of the wave, 2) water depth, 3) waveheight, and 4) angle of incidence. By searching the parameter space, the conditions under which both the maximum onshore and offshore force occur can be found. It should be noted that we refer in the following section to the depth-integrated force per unit length as derived in Section 6.1.

1) Variation with phase

Figure 7 shows a typical variation of force with phase, $\chi$. The maximum onshore force in this case occurs under the crest $\chi=0$ and the maximum offshore force occurs at the trough $\chi=\pi$. Figure 8 shows a case where the water is deeper and the wave higher. In this case the maximum onshore force does not occur at the crest, but the force increases from $\chi=0$ to about $\chi=0.8$ and then rapidly deceases. Again the maximum offshore force occurs at $\chi=\pi$.

In general an increase in $\theta$ or a decrease in depth will move the maximum onshore force towards the crest. An increase in wave steepness has the effect of moving the maximum onshore force away from the crest. The maximum offshore force always occurs at the trough. 


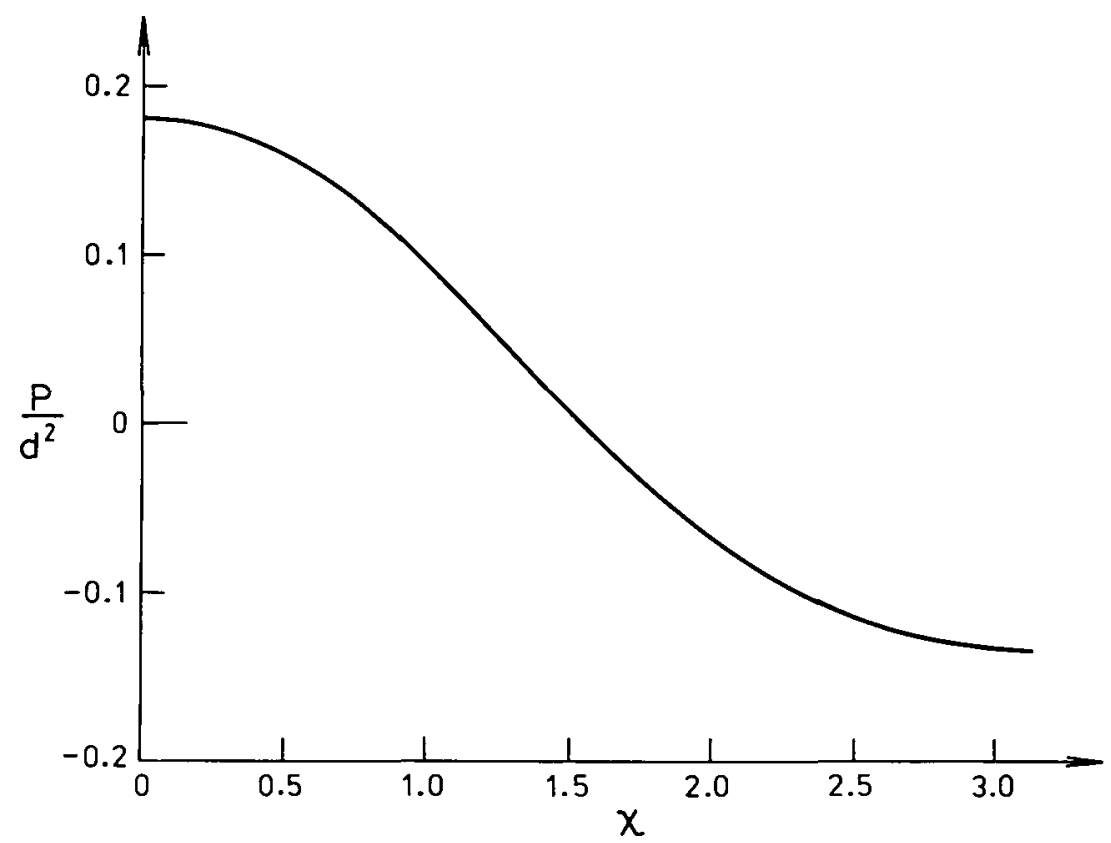

Figure 7. Variation of depth integrated force per unit length with phase, $\chi$, for $d=0.8, \theta=0^{\circ}$ and $h=0.16$.

For standing waves the second harmonic term at second order in the series expansion for the pressure does not decay with depth but remains finite down to the fluid bed. So for near-standing waves in deep water the depth-integrated onshore force contains a large second harmonic contribution at second order. This results in secondary humps appearing on a plot of pressure versus phase (see Goda [9]) and hence explains the result that the maximum onshore force can occur away from the crest.

\section{2) Variation with angle and depth}

Figure 9 shows the maximum onshore and offshore wave force for $d=0.8$ and $h=0.16$. The maximum onshore force occurs under the crest for all $\theta$, and shows a small gradual increase as $\theta$ varies from $0^{\circ}$ to $90^{\circ}$. The maximum offshore force stays relatively constant between $\theta=0^{\circ}$ and $\theta=50^{\circ}$, and then decreases to a minimum. In fact there is a slight increase in force between $\theta=0^{\circ}$ and about $\theta=30^{\circ}$ but it is less than a $1 \%$ variation. The onshore force is greater than the offshore force in this case. Figure 10 is for a wave with $d=5$ and $h=0.28$. This wave in deeper water shows the same general trend as Figure 9 with the following differences: 1) the maximum force does not occur under the crest for $\theta \leqslant 15^{\circ}$ but 


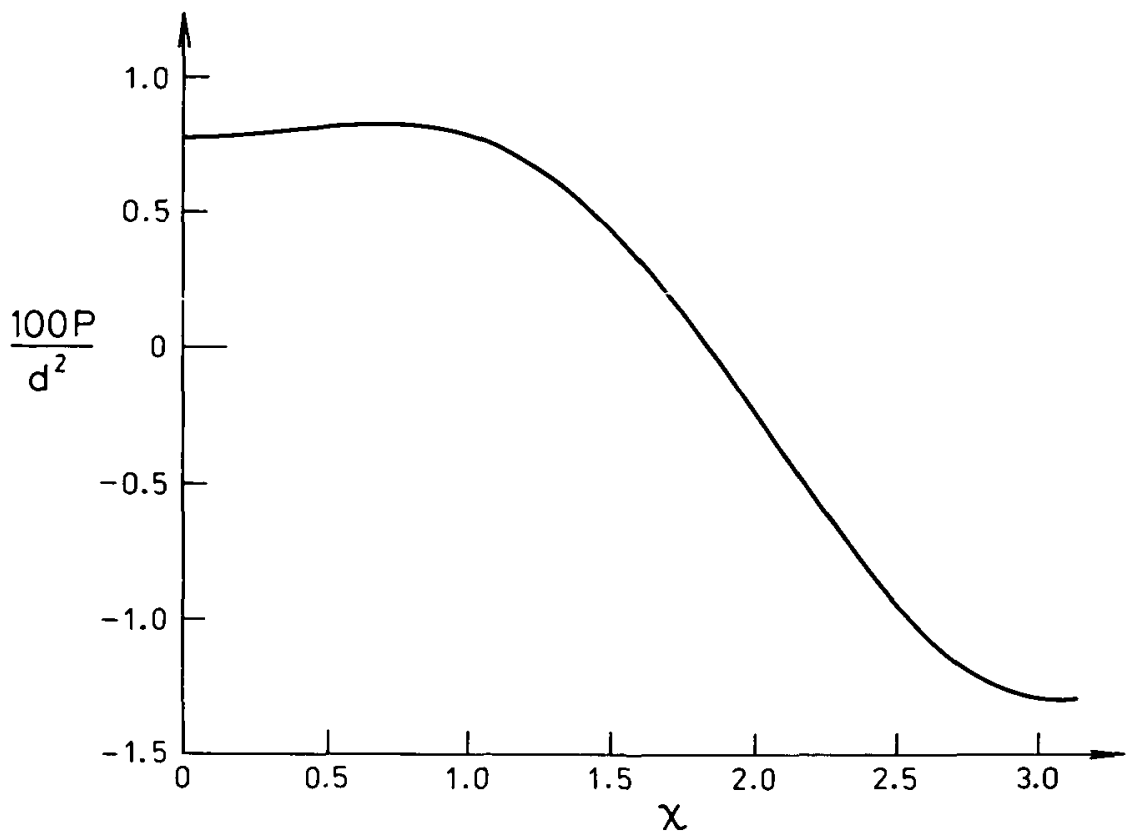

Figure 8. Variation of depth integrated force per unit length with phase, $\chi$, for $d=5.0, \theta=10^{\circ}$ and $h=0.28$.

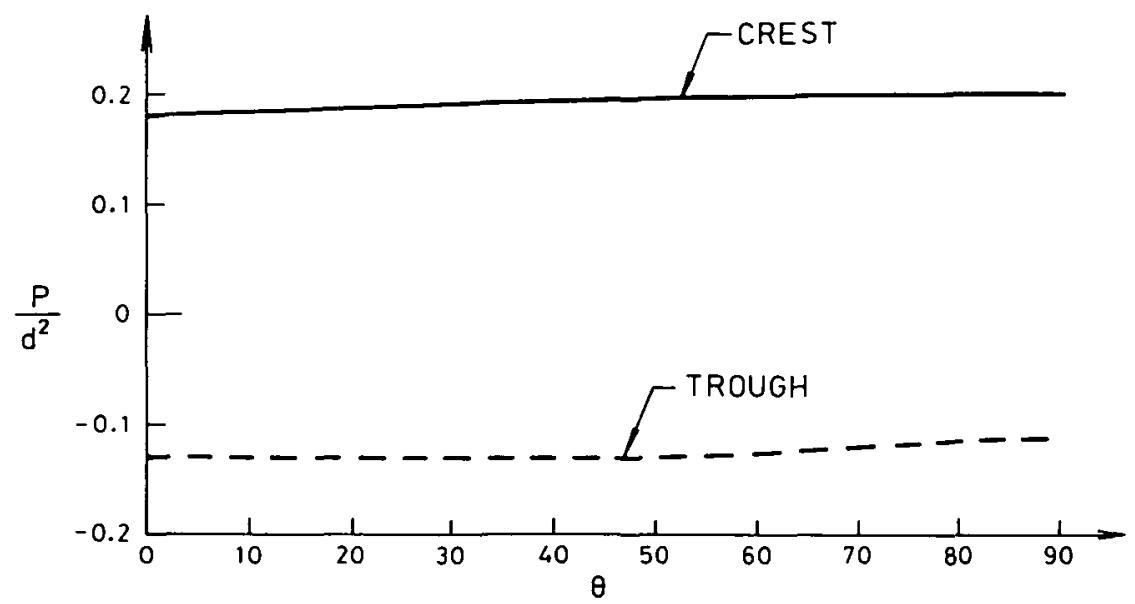

Figure 9. Variation of depth integrated force per unit length with $\theta$ for $d=0.8$ and $h=0.16$. Shown is the force at the crest and the trough. 


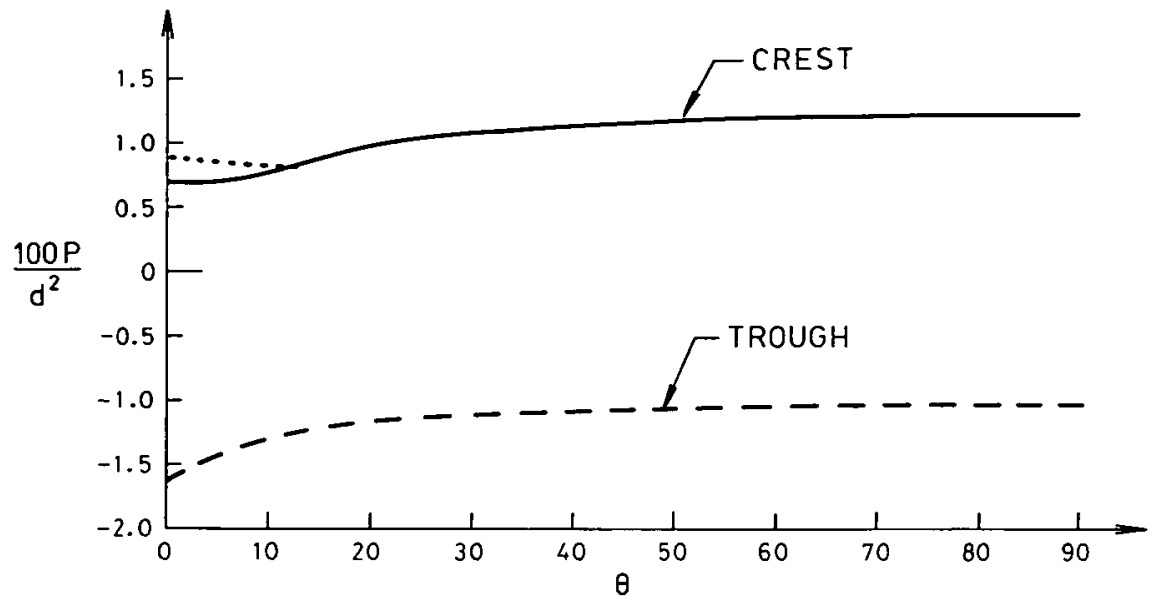

Figure 10. Variation of depth integrated force per unit length with $\theta$ for $d=5.0$ and $h=0.28$. Shown is the force at the crest (-), the trough $(---)$ and the intermediate maximum $(\cdots)$.

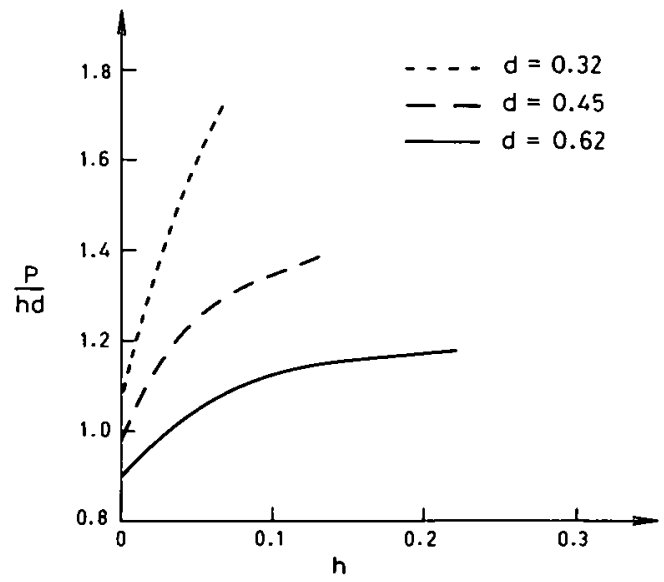

Figure 11. Maximum onshore depth integrated force per unit length versus waveheight near the progressive wave limit $\left(\theta=80^{\circ}\right)$ for $d=0.32, d=0.45$ and $d=0.62$.

at about $\chi=0.8$; 2) the variation of force with $\theta$ is more marked; 3) the maximum offshore force occurs at $\theta=0^{\circ}$; and 4) the maximum offshore force is now greater than the maximum onshore force. Figure 11 shows the force near the progressive wave limit $\left(\theta=80^{\circ}\right)$ for $d=0.62,0.45$ and 0.32 . The general trend is that all forces increase as $d$ decreases.

This leads to the conclusion that the maximum onshore force will occur for an oblique wave in shallow water for a wave of large height (near the maximum possible). As an indication of how much greater the onshore force can be, a wave 
approaching the wall with $\theta=80^{\circ}, d=0.32$ and $h=.07$ will subject the wall to a $21 \%$ greater force than a standing wave of the same height and depth. The maximum offshore force will occur for a high wave in shallow water with $\theta \approx 30^{\circ}$, but the difference in force between this wave and the standing wave is negligible. As the maximum offshore force is less than the maximum onshore force in shallow water, the maximum onshore force is the main design criterion.

The result that the maximum onshore furce is greater for a wave striking the wall at an oblique angle rather than for a normal approach is not intuitively expected and needs some explanation. The increase in force as $\theta$ increases is due to the term $\omega \phi_{X}$ in Bernoulli's equation. This term represents the local acceleration of fluid particles at the wall. This increase in acceleration as $\theta$ increases seems to be due to the fact that the waves become slightly more peaked in the $X$-direction as $\theta$ increases.

\subsection{The average force}

So far we have considered the fluctuating forces on the wall; now we shall compare these with the average force on the wall. Equation (6.5) becomes

$$
F^{\prime \prime}=\sum_{r=2}^{\infty} h^{r} F_{r 0} \quad \text { per unit length }
$$

as all the other terms are oscillatory. Observe that the average force is of order two in waveheight while the fluctuating force is of order one. Therefore the average force is much smaller than the fluctuating forces.

The average depth-integrated force is onshore, and the maximum occurs for the standing wave case rather than for the oblique wave which was the case for fluctuating forces. The reason that the maximum average force decreases as $\theta$ increases is that the contribution from the dynamic term in Bernoulli's equation, $-\frac{1}{2} \rho|\nabla \phi|^{2}$, becomes larger in magnitude. Now

$$
|\nabla \phi|^{2}=p^{2} \phi_{X}^{2}+\phi_{y}^{2}
$$

and the average force contains only the phase independent terms so the magnitude of the above term grows due to increasing $p$ as $\theta$ increases. Physically, this is due to the velocity in the $x$-direction increasing from zero as $\theta$ increases from $0^{\circ}$.

\section{Conclusion}

Using a 35 th order perturbation expansion we have made estimates of various properties of short-crested waves in water of finite depth. For example, the maximum wave height for $d=0.8$ has beeniestimated to an error of about $3 \%$ except where harmonic resonance adversely affects the results. 
As a practical application the pressure exerted by waves being reflected by a seawall is examined. It is found that the maximum force occurs in the case of a steep wave in shallow water approaching the wall obliquely. As an example, a wave with $d=0.32$ and $h=0.07$ that approaches the wall obliquely $\left(\theta=80^{\circ}\right)$ exerts a $21 \%$ greater force than an equivalent standing wave.

Further investigation could be done into standing waves in finite depth since little work has been done in this area. However, shallow water would certainly present difficulties due to the large numbers of harmonic resonances that occur for $d \leqslant 0.5$.

\section{References}

[1] C. M. Bender \& S. A. Orszag, Advanced Mathematucal Methods for Scientists and Engineers (McGraw-Hill, New York, 1978).

[2] P. J. Bryant, "Two-dimensional periodic permanent waves in shallow water", J. Fluid Mech. 115 (1982) 525-532.

[3] J. E. Chappelear, "On the description of short-crested waves", Beach Erosion Board, U.S. Army Corps Engrs, Tech. Memo 125, 1961.

[4] B. Chen \& P. G. Saffman, "Steady gravity-capillary waves on deep water-1. Weakly nonlinear waves", Stud. Appl. Math. 60 (1979) 183-210.

[5] E. D. Cokelet, "Steep gravity waves in water of arbitrary uniform depth", Philos. Trans. Roy. Soc. Lond. A 286 (1977) 183-230.

[6] P. Concus, "Standing capillary gravity waves of finite amplitude: corrigendum", J. Fluid Mech. 19 (1964) 264-266.

[7] J. D. Fenton, "Short-crested waves and the wave forces on a wall", J. Water, Port, Coast,, and Ocean Eng. 111 (1985) 693-718.

[8] R. A. Fuchs, "On the theory of short-crested oscillatory waves", Gravity Waves, U. S. Nat. Bur. Stand. Circular 521 (1952) 187-200.

[9] Y. Goda, "The fourth order approximation to the pressure of standing waves", Coast. Eng. in Japan 10 (1967) 1-11.

[10] J. R. C. Hsu, Y. Tsuchiya \& R. Silvester "Third-order approximation to short-crested waves", J. Fluid Mech. 90 (1979) 179-196.

[11] L. F. McGoldrick, "On Wiltons ripples: a special case of resonant interactions", J. Fludd Mech. 42 (1970) 193-200.

[12] L. F. McGoldrick, "On the rippling of small waves: a harmonic nonlinear nearly resonant interaction", $J$. Fluid Mech. 52 (1972) 725-751.

[13] A. J. Roberts, "The behavior of harmonic resonant steady solutions to a model differential equation", Q. J. Mech. Appl. Maths. 34 (1981) 287-310.

[14] A. J. Roberts \& D. H. Peregrine, "Notes on long-crested waves", J. Fluid Mech. 135 (1983) 323-335.

[15] A. J. Roberts \& L. W. Schwartz, “The calculation of nonlinear short-crested gravity waves”, Phys. Fluids 26 (1983) 2388-2392.

[16] A. J. Roberts, "Highly nonlinear short-crested waves", J. Fluid Mech. 135 (1983), 301-321.

[17] J. W. Rottman, "Steep standing waves at a fluid interface", J. Fluid Mech. 124 (1982) 283-306.

[18] L. W. Schwartz \& A. K. Whitney, "A semi-analytic solution for nonlinear standing waves", $J$. Fluid Mech. 107 (1981) 147-171.

[19] I. Tadjbakhsh \& J. B. Keller, "Standing surface waves of finite amplitude", J. Fluid Mech. 8 (1960) 442-451. 